\title{
A TB40/E-derived human cytomegalovirus genome with an intact US-gene region and a self-excisable BAC cassette for immunological research
}

Kerstin Laib Sampaio ${ }^{1}$, Anja Weyell ${ }^{1}$, Narmadha Subramaniann ${ }^{1}$, Zeguang $\mathrm{Wu}^{1,{ }^{\dagger}}$, and Christian Sinzger ${ }^{1}$ 'Institute of Virology, University of Ulm, Ulm, Germany

†Present address: Memorial Sloan Kettering Cancer Center, New York, NY

BioTechniques 63:205-214 (November 2017) doi 10.2144/000114606

Keywords: HCMV, TB40/E, self-excisable BAC cassette, US genes, MHC-I

Supplementary material for this article is available at www.BioTechniques.com/article/114606.

For immunological research on the human cytomegalovirus (HCMV), a virus that combines the broad cell tropism of clinical isolates, efficient replication in cell culture, the complete set of MHC-I modulator genes, and suitability for genetic engineering is desired. Here, we aimed to generate a genetically complete derivative of HCMV strain TB40/E as a bacterial artificial chromosome (BAC) with a self-excisable BAC cassette. The BAC cassette was inserted into the US2-US6 gene region (yielding TB40-BAC $\mathrm{KL}_{\mathrm{T}}$ ), relocated into the UL73/UL74 region with modifications that favor excision of the BAC cassette during replication in fibroblasts, and finally the US2-US6 region was restored, resulting in $\mathrm{BAC}$ clone TB40-BAC $\mathrm{KL}_{7}-\mathrm{SE}$ When this BAC clone was transfected into fibroblasts at efficiencies $>0.1 \%$, replicating virus that had lost the BAC cassette appeared within 2 weeks after transfection, grew to high titers, and displayed the broad tropism of the parental virus. The degree of MHC-I down-regulation by this virus was consistent with functional restoration of US2-US6. To enable detection of infected cells by flow cytometry, an enhanced green fluorescent protein (EGFP)-expression cassette was inserted downstream of US34A, yielding the fluorescent virus RV-TB40-BAC $\mathrm{KL7}_{7}-\mathrm{SE}-\mathrm{EGFP}$.

Human cytomegalovirus (HCMV) belongs to the betaherpesvirus family and is a widespread human pathogen that exhibits lifelong persistence in the host after infection. HCMV can lead to severe, life-threatening diseases in immunocompromised patients and is the most common cause of intrauterine infections during pregnancy, occasionally with fatal outcomes (1). Understanding the interplay of HCMV with its host is of major interest (e.g., virus-induced downregulation of immune recognition). Development of infectious bacterial artificial chromosomes (BACs) has greatly advanced HCMV research by facili- tating the generation and analysis of viral mutants. However, to date, the application of HCMV-derived BACs, especially in immunological research, is still limited by the fact that available BACs either lack immunologically relevant genes or do not produce high-titer progeny with a broad cell tropism.

Previously, we created a BAC clone of the HCMV strain TB40/E (TB40-BAC4) that combines the high-titer production of lab strains with the extended cell tropism of recent isolates (2). However, TB40-BAC4 lacks the MHC-I downregulating genes US2, US3, and US6 due to insertion of the BAC cassette in the US1-US6 region. Therefore, we sought to generate a TB40/E-derived BAC containing the full set of viral genes. Simple reinsertion of missing genes into the existing TB40-BAC4 is not an option as the resulting genome would likely exceed the packaging limit, and the resulting virus might be prone to unwanted second-site deletions due to a loss of replicative fitness (3). To overcome this problem, we decided to engineer the genome in a way that the BAC cassette would be excised seamlessly from the genome during virus reconstitution. This not only avoids the issue of an oversized genome, but also solves the

\section{METHOD SUMMARY}

We provide a new human cytomegalovirus (HCMV) bacterial artificial chromosome (BAC), containing an intact US gene region and a self-excisable BAC cassette, that grows to high titers and displays the extended phenotype for epithelial and endothelial cells. The presence of all known MHC-I modulator genes facilitates HCMV research, particularly on immunological topics. A green fluorescent version of this virus simplifies analysis by flow cytometry. 
problem that HCMVs retaining the BAC cassette during virus propagation tend to acquire mutations around the BAC vector sequence (4).

\section{Materials and methods}

Cells and viruses

Primary human foreskin fibroblasts (HFFs) were cultivated in MEM supplemented with GlutaMAX (Life Technologies GmbH, Darmstadt, Germany), $5 \%$ fetal calf serum (FCS) (PAN Biotech GmbH, Aidenbach, Germany), $0.5 \mathrm{ng} / \mathrm{mL}$ basic fibroblast growth factor (bFGF) (Life Technologies), and $100 \mu \mathrm{g} / \mathrm{mL}$ gentamicin. Human umbilical vein endothelial cells (HUVECs) were seeded onto gelatin-coated vessels and maintained in RPMI1640 medium (Life Technologies) supplemented with $10 \%$ HCMV-seronegative human serum, 50 $\mu \mathrm{g} / \mathrm{mL}$ endothelial cell growth supplement (BD Biosciences, Heidelberg, Germany), 5 units/mL heparin (Sigma-Aldrich, Taufkirchen, Germany), and $100 \mu \mathrm{g} / \mathrm{mL}$ gentamicin (Life Technologies).

The original strain TB40/E (5) was again plaque-purified, and an endotheliotropic clone selected by phenotypic testing, designated $40 \mathrm{E}$, then served as the basis of our experiments. The detailed construction of BAC clones TB40-BAC ${ }_{K L L}$, TB40-BAC $\mathrm{KL}_{\mathrm{T}} \mathrm{SE}$ and TB40-BAC $\mathrm{KL}_{7}$-SEEGFP is provided in the Supplementary Material. Virus stocks were generated by centrifugation of supernatants at 2800 $\times \mathrm{g}$ for $10 \mathrm{~min}$ to remove cellular debris and stored at $-80^{\circ} \mathrm{C}$. For infections, cells were pre-incubated for 30 min with MEM containing 5\% FCS and then incubated for $2 \mathrm{~h}$ with cell-free virus preparations. Finally, medium was replaced with the respective cell culture medium, and cells were further incubated.

\section{Immunofluorescence}

For the detection of viral immediate-early (IE) antigens pUL122/123, cells were fixed with $80 \%$ acetone and incubated sequentially with primary antibody E13 (Cat. \#11-003; Argene/Biomerieux, Nürtingen, Germany; RRID: AB_2716294) and secondary antibody Cy3-goat antimouse IgG $F\left(a b^{\prime}\right)_{2}$ (Cat. \#115-166-062; Jackson ImmunoResearch, West Grove, PA; RRID: AB_2338703). To detect natural enhanced green fluorescent protein (EGFP) signals in combination with IE antigens, cells were fixed with $4 \%$ paraformaldehyde for $10 \mathrm{~min}$ at room temper-

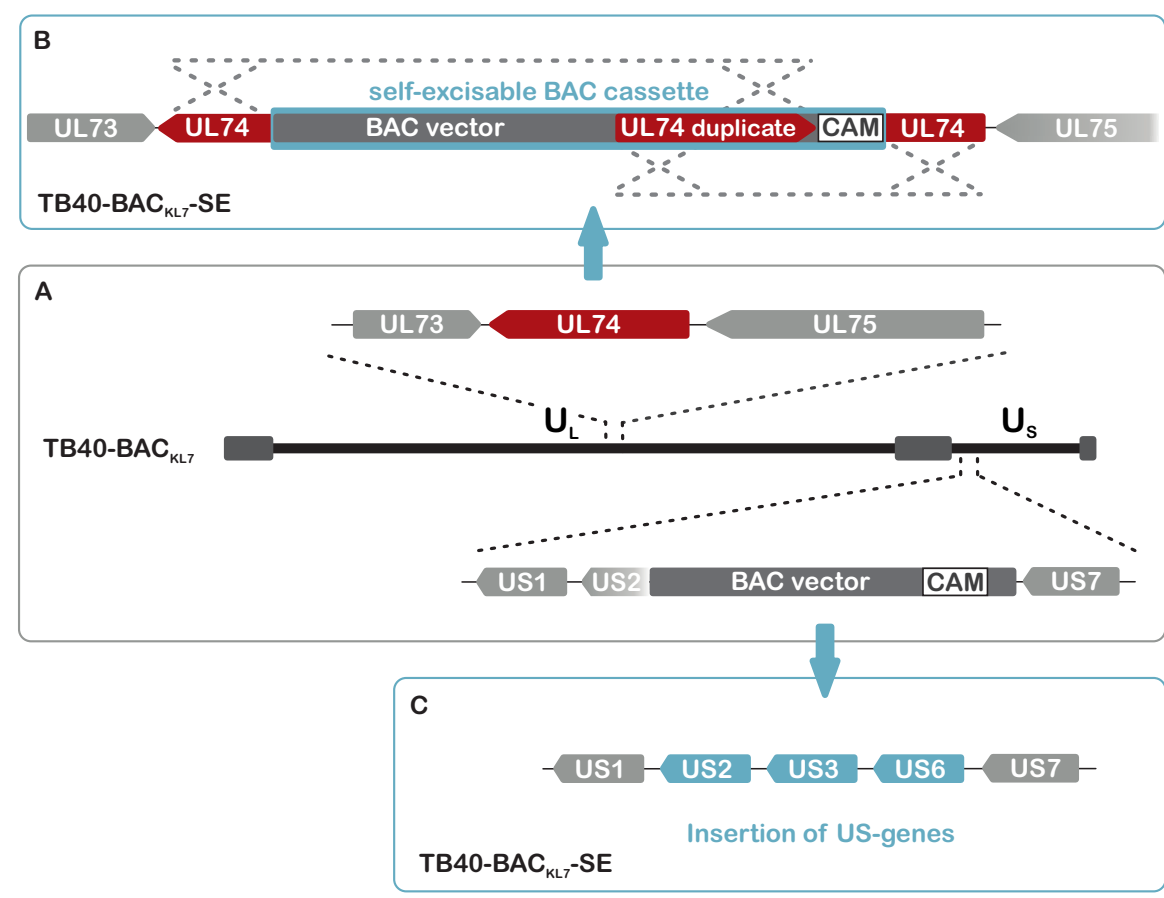

Figure 1. Schematic representation of the TB40-BAC ${ }_{K L 7}$ and TB40-BAC ${ }_{K L 7}-S E$ genomes. (A) TB40$B A C_{K L 7}$ was generated by insertion of the vector cassette to replace the genes US2, US3, and US6 within human cytomegalovirus (HCMV) strain TB40/E. (B) Representation of the self-excisable vector cassette in TB40-BAC $\mathrm{KL}_{7}$ SE The vector cassette was relocated into the middle of the UL74 open reading frame and a UL74 homology duplicate was inserted in the vector cassette. The two intra- or intermolecular recombination events leading to vector excision from the upstream half of the UL74 ORF toward the inverted half of the UL74 duplicate and the downstream part of the UL74 ORF toward the second half of the inverted UL74 duplicate are indicated by dotted lines. (C) The US gene region was restored by reinsertion of genes US2, US3, and US6, replacing the primary vector sequence and resulting in the final TB40-BAC $\mathrm{KL}_{7}$ SE genome. CAM: chloramphenicol resistance gene. A comprehensive schematic representation of the entire cloning procedure is provided in Supplementary Figure S1.

ature, permeabilized for 10 min with PBS containing $10 \%$ sucrose, $1 \%$ FCS, and $0.5 \%$ Nonidet P40 (all purchased from Sigma-Aldrich) and incubated with antibodies as described above.

\section{Virus reconstitution}

BAC DNA was isolated using the NucleoBond Xtra Midi Kit (MachereyNagel, Düren, Germany) and transfected into HFFs in 6-well plates by calcium phosphate transfection (MBS Transfection Kit; Agilent, Waldbronn, Germany), as described in the Supplementary Protocol. To monitor the process of virus reconstitution, a portion of the culture $\left(1 \times 10^{5}\right.$ cells) was removed when cultures were passaged, seeded into 24-well plates and stained for viral IE-antigens 3-4 $\mathrm{h}$ after adherence. Cells were counted, and the percentage of IE-positive cells was calculated.

To assess individual virus passages for the absence of the BAC vector cassette and the presence of an intact UL74 ORF, DNA from supernatants was purified using the DNeasy Blood \&
Tissue Kit (Qiagen, Hilden, Germany), and PCR was performed using chloramphenicol resistance gene- (CAM) or UL74-specific primers. The resulting UL74 amplification products were purified with the NucleoSpin PCR Clean-up Kit (Macherey-Nagel) and checked by Sanger sequencing (GATC, Konstanz, Germany).

\section{Whole-genome sequencing}

HFFs were infected with reconstituted virus RV-TB40-BAC ${ }_{K L 7}-S E$ from the first cell-free passage after transfection at a multiplicity of infection (MOI) of 10. Viral DNA was extracted from this culture at 3 days post-infection (p.i.) using the method of Hirt $(6,7)$. BAC-DNA from bacterial cultures of TB40-BAC $\mathrm{KL}_{7}-\mathrm{SE}$ was isolated using the NucleoBond Xtra Midi Kit. Both DNA from the BAC clone and from the reconstituted virus were sent to an external sequencing facility (SEQ-IT GmbH u. Co. KG, Kaiserslautern, Germany), where indexed libraries were generated with the Illumina TruSeq DNA PCR-free Kit and subse- 


\section{Introducing ali-Q ${ }^{\mathrm{TM}}$}

The first-ever

aliquoting pipet controller

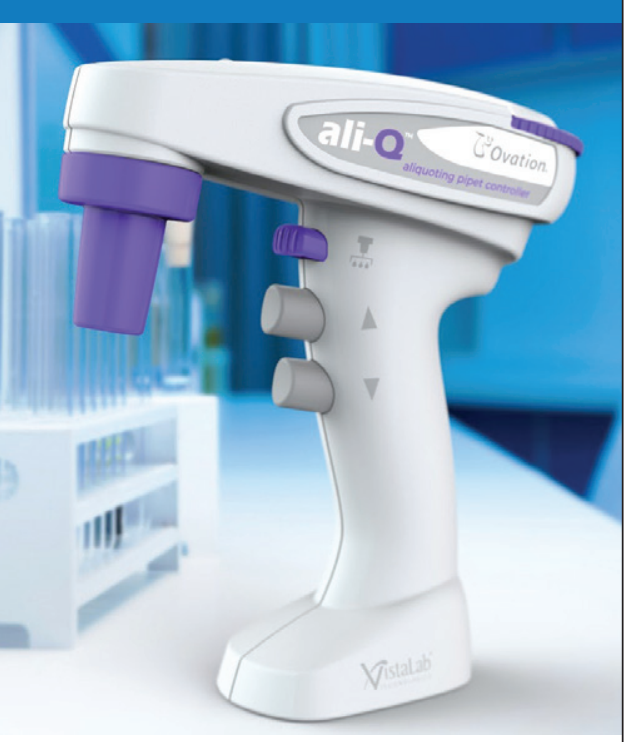

\section{One-button aliquoting}

Get accurate and precise aliquots without the need to "eyeball" the meniscus.

- Faster aliquoting with the press of one button.

- Also aspirates and dispenses like other pipet controllers.

- Uses any size or brand serological pipet.

\section{Use VistaLab ${ }^{T M}$ Wobble-not $^{\text {TM }}$}

serological pipets with ali- $Q$ for the ideal pipetting system.
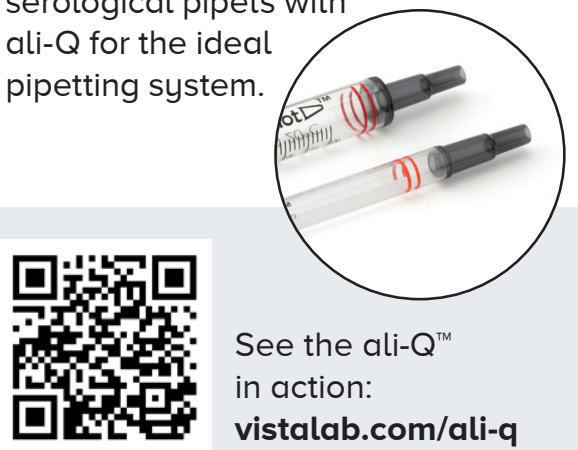

See the ali- $Q^{\text {TM }}$ in action: vistalab.com/ali-q

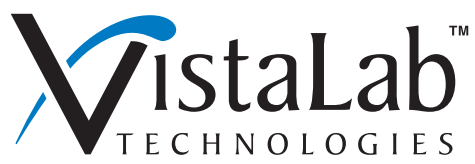

Think Differently. Feel Better.

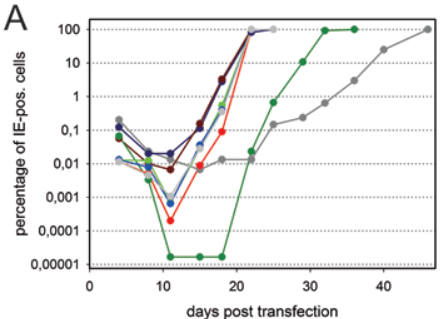

B

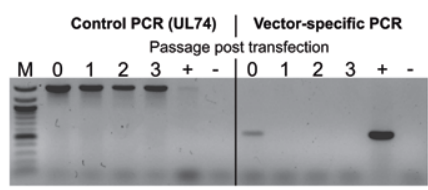

C

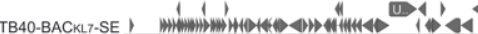

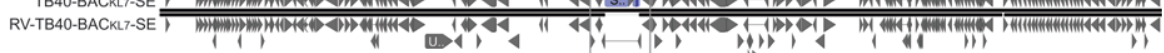

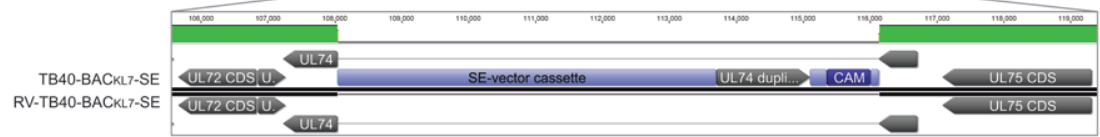

Figure 2. TB40-BAC ${ }_{\mathrm{KL}}-\mathrm{SE}$ can be successfully reconstituted, grows to high titers, and efficiently infects endothelial cells. (A) BAC DNA from TB40-BAC ${ }_{K 17}-\mathrm{SE}$ was isolated and transfected into human foreskin fibroblasts (HFFs). Eight individual transfections were followed over time. At each time point post-transfection, a portion of the cells was stained for viral immediate early (IE) antigen, and the percentage of IE-positive cells was calculated. The majority of transfected cultures reached $100 \%$ infection within 3 weeks. (B) After reconstitution, consecutive cell-free virus passages were tested for the presence of bacterial artificial chromosome (BAC) vector sequences by PCR. Supernatants of infected cultures were harvested 5 days post-infection (p.i.), viral DNA was isolated, and PCR was conducted with BAC vector-specific primers. UL74-specific primers served as a control. Vector-specific sequences were already undetectable after one cell-free passage, indicating efficient removal of the BAC cassette. Positive control (+) BAC DNA was used for transfection. (C) BAC DNA from TB40-BAC ${ }_{K 17}-S E$ and viral DNA from the respective reconstituted virus RV-TB40-Bac ${ }_{K 17}-S E$ were subjected to next-generation sequencing (NGS). The alignment of both sequences revealed the correct removal of the vector cassette in the reconstituted virus RV-TB40-Bac ${ }_{K 17}-S E$ and showed the correct integrity of the genome. Only two single-base pair changes could be detected, neither of them leading to an amino acid change (yellow lines within the green identity column). The zoomed region of the BAC vector cassette is indicated by a gray box. (D) The reconstituted virus of RV-TB40$\mathrm{BaC}_{K 17}$-SE was compared with the parental TB40/E strain (40E) in a multi-step growth curve in HFFs infected at a multiplicity of infection (MOI) of 0.03. Error bars represent standard error of the mean (SEM) from logarithmic titer values titrated in duplicate. (E) Phenotype of RV-TB4O-BAC $\mathrm{KLT}_{-}-\mathrm{SE}$ and the parental $40 \mathrm{E}$ strain in infection of endothelial cells. Three independently generated supernatants for each strain were serially diluted and used to infect HFFs and human umbilical vein endothelial cells (HUVECs). At $24 \mathrm{~h}$ p.i., cells were stained for viral IE antigen, and nuclei were counterstained with 4',6-diamidino-2-phenylindole (DAPI). The fraction of infected cells was determined for each dilution step and used to calculate the apparent MOIs in HFFs and HUVECs. The ratio $\mathrm{MOI}_{\mathrm{HUVEC}} / \mathrm{MOI}_{\mathrm{HFF}}$ characterizes the tropism of the respective virus. Error bars represent standard error of the mean (SEM).

quently sequenced on an Illumina MiSeq instrument. The resulting reads were analyzed with the Geneious 6.1.8 software (8). For both samples, de novo assemblies were performed with standard settings, and the resulting contigs were assembled against the sequences of TB40-BAC4 (GenBank \#EF999921) and TB40/E clone Lisa (\#KF297339). The remaining unassembled contigs were checked for the presence of additional HCMV sequences and, if present, included in the data set. These contigs were used to scaffold a hybrid sequence where gaps were filled with TB40-BAC4 sequences. Individual Illumina reads were mapped to this hybrid sequence [coverage: $842.3 \pm 126.3$ reads (mean \pm SD)], and unassembled reads were checked for the absence of HCMV sequences. Finally, the respective consensus sequences were extracted, manually corrected, and annotated in reference to the annotations of the strain Merlin (\#NC_006273) or TB40/E clone Lisa. The sequence of the BAC clone TB40-BAC KL $_{7}$ SE was deposited in NCBI GenBank (\#MF871618). 


\section{Growth curves}

HFFs in 24-well plates were infected with cell-free virus supernatants at an $\mathrm{MOI}$ of 0.03 (multi-step growth curves) or an $\mathrm{MOI}$ of 5 (single-step-growth curves) for $2 \mathrm{~h}$. Cultures were washed with MEM to remove residual virus and cultured for 7 or 14 days, respectively. Supernatants were removed from infected cultures at the indicated time points and replaced by fresh medium. Samples were centrifuged at $2800 \times \mathrm{g}$ for $10 \mathrm{~min}$ and stored at $-80^{\circ} \mathrm{C}$ prior to determination of the infectious titer by limiting dilution assay as described previously (9).

\section{Fluorescence-activated cell sorting} HFFs in 6-well plates were infected with the respective virus preparation for 2 $h$, followed by a medium exchange. At the indicated time points, cultures were incubated in prewarmed $0.04 \%$ EDTA in PBS (Versene; Biochrom, Berlin, Germany) for $10 \mathrm{~min}$ to detach cells, washed twice in PBS containing 5\% FCS, and incubated on ice with phycoerythrin (PE)-conjugated anti-HLA ABC antibody (Cat. \#21159034; W6/32; Immunotools, Friesoythe, Germany; RRID: B_2629494) or the respective isotype control. To analyze MHC-I surface levels on EGFPexpressing cells, cells were detached with citric saline buffer (135 mM potassium chloride, 15 mM sodium citrate) (SigmaAldrich), incubated with an allophycocyanin (APC)-conjugated anti-HLA ABC antibody (Cat. \#1159036, W6/32; Immunotools; RRID: AB_2716295) or the respective isotype control, and analyzed in a FACS Calibur (BD Biosciences). Data sets were processed with FlowJo (FlowJo, LLC, Ashland, OR).

\section{Statistical analyses}

Data sets were analyzed by two-wayANOVA using the built-in data analysis function of SigmaPlot (Systat Software Inc., San Jose, CA) to test for significant differences between the various conditions. If ANOVA indicated significant differences between groups, post-hoc analyses by $t$-test were performed to identify which of the groups differed from the others. Only the $P$ values of post-hoc tests are mentioned in the results section.

\section{Results and discussion}

As a starting point, we generated a new BAC clone of strain TB40/E with the objective of obtaining a more intact genome as compared with TB40-BAC4, which had accidentally lost a 3-kb region (IRS1-US1) adjacent to the BAC cassette. For this, the method of Borst et al. (7) was applied to generate $\mathrm{TB} 40-\mathrm{BAC}_{\mathrm{KL} 7}$, which
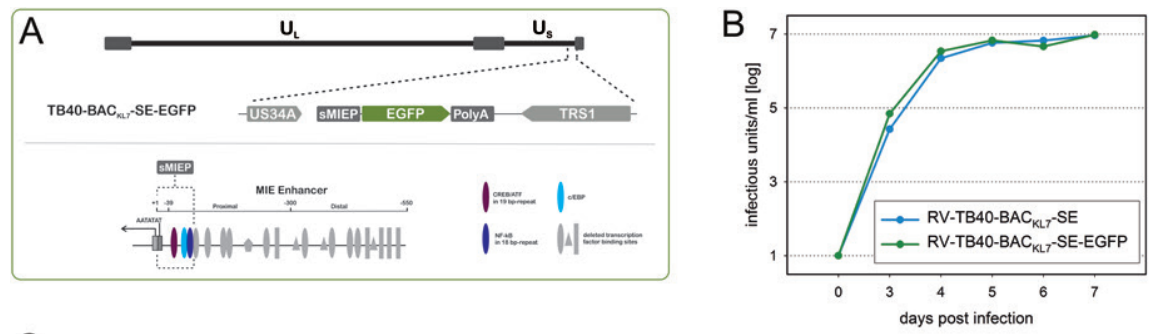

C
$2 \mathrm{~h} \mathrm{p.i.}$

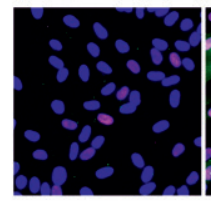

4 h p.i.

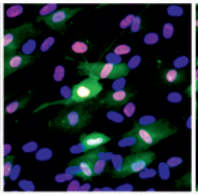

$6 \mathrm{~h}$ p.i.

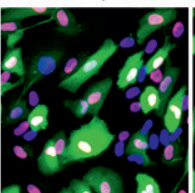

8 h p.i.

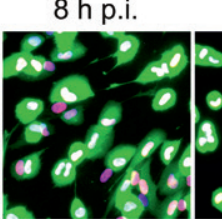

24 h p.i.

72 h p.i.
Figure 3. Insertion of an enhanced GFP (EGFP) expression cassette into TB40-BAC ${ }_{\mathrm{KLT}}-\mathrm{SE}$. (A) Schematic representation of the TB40-BAC $\mathrm{KL}_{-}$-SE-EGFP genome with the EGFP expression cassette located downstream of the US34A gene locus. In the lower part of the panel, the major immediate early promotor (MIEP) with individual transcription factors is depicted. Removal of $459 \mathrm{bp}$ of the MIE enhancer region resulted in the shortened version of the promotor, designated as SMIEP (indicated by a dotted gray box; length of the remaining promotor corresponds to $96 \mathrm{bp}$ until the TATA box). Promotor scheme is shown as depicted by Keller et al. (34) (B) Single-step growth curve of RV-TB40-BAC $\mathrm{KLI}_{7}$-SE-EGFP in comparison to the parental RV-TB40$B C_{K L}-S E$ in human foreskin fibroblasts (HFFs) infected at a multiplicity of infection (MOI) of 5. (C) EGFP expression kinetics in HFFs infected with $\mathrm{BAC}_{\mathrm{KL}}$-SE-EGFP at an $\mathrm{MOI}$ of 50 . Cells were fixed with paraformaldehyde at the indicated time points, stained for viral immediate early (IE) antigen proteins pUL122/123 (Cy3), and pictures were taken of the natural EGFP signal together with red Cy3 signal. Nuclei were counterstained with 4',6-diamidino-2-phenylindole (DAPI). showed correct insertion of the BAC cassette within the targeted US2-US6 region. Sequencing confirmed consistency between TB40-BAC KL7 $_{7}$ and the parental 40E virus, which showed that both genomes contain an intact US1 gene but a short IRS1 version as compared with the HCMV consensus sequence (676 bp deleted, resulting in an IRS1 protein 335 amino acids-long due to a premature stop codon).

To generate a self-excisable version of TB40-BAC $\mathrm{KL}_{\mathrm{K}}$, we used the method developed by Tischer et al. (10-12) , which uses inverted viral sequence duplications within the vector cassette that are homologous to the regions flanking the BAC vector cassette (Figure 1). Vector excision relies on recombination between these duplicated homologies within eukaryotic cells upon transfection. In the original method, an essential gene was used as the site for BAC vector insertion to provide a growth advantage for viruses that eliminate vector sequences and thereby repair the disrupted gene. We chose the nonessential gene UL74 for this purpose, as disruption of this reading frame in HCMV lab strains substantially decreased cell-free infectivity (13-16). Thus, insertion of an 8-kb vector cassette into this gene should result in a predominantly cellassociated virus and establish a selection pressure toward viruses that have excised the vector cassette. Surprisingly, it was not possible to reconstitute virus from DNA of an intermediate construct that harbored the BAC cassette within UL74 but did not yet contain the homology duplicates necessary for vector excision. This intermediate construct corresponds to an UL74-deletion mutant and should have grown with the respective cell-associated phenotype, since the BAC cassette remains in the genome. Most probably, introduction of the large vector cassette additionally affected the transcription of the neighboring essential UL73 gene, as its transcript is spliced over the UL74 ORF toward a splice acceptor site downstream of UL74 (17). However, we continued with our cloning strategy, as Tischer et al. had reported successful reconstitution of viruses from lethal introductions of vector cassettes $(10,11)$. After insertion of the UL74 homology duplicate into the vector cassette and restoration of the US gene region, the final $\mathrm{TB} 40-\mathrm{BAC}_{\mathrm{KL} 7}-\mathrm{SE}$ genome could be successfully reconsti- 
tuted in HFFs using a calcium phosphatebased transfection method. For reliable virus reconstitution, the combination of transfection efficiencies $>0.1 \%$ and the usage of synchronized HFFs by serum starvation turned out to be essential, as transfection during the S phase of the cell cycle may facilitate homologous recombination within eukaryotic cells (18). A detailed protocol is included in the Supplementary Material. Using this method, an increase in the number of infected cells could be detected as early as 8 days post-transfection, and the majority of cultures reached $100 \%$ infected cells within 3 weeks of transfection (Figure 2A). Viruses from the supernatants of transfection cultures were further propagated in HFFs and monitored for the presence of vector sequences by PCR. Vector-specific sequences were already hardly detectable in supernatants of the original transfection culture and became undetectable after only one further passage with cell-free virus, indicating that only viruses that have excised their vector cassette are replicationcompetent (Figure 2B). The absence of the BAC vector was confirmed by wholegenome sequencing (next-generation deep sequencing) of DNA extracted from reconstituted virus $\mathrm{RV}$-TB40-BAC $\mathrm{BL}_{\mathrm{K}}-\mathrm{SE}$ after the second cell-free passage and compared with the sequence obtained from BAC-DNA of TB40-BAC $\mathrm{KL}_{7}-\mathrm{SE}$. This comparison not only confirmed the previous finding that the vector was excised seamlessly from the genome but also proved the integrity of the whole genome after reconstitution (Figure 2C). Only two single base-pair exchanges could be detected, one located within UL57 (a silent mutation not leading to an amino acid exchange) and one within the non-coding US/UL-junction region. To ensure that correct removal of vector sequences can be reliably achieved, PCR products amplified from the UL74 genes of 10 independent virus reconstitutions were checked by sequencing, and the expected UL74 sequence was confirmed for each (data not shown).

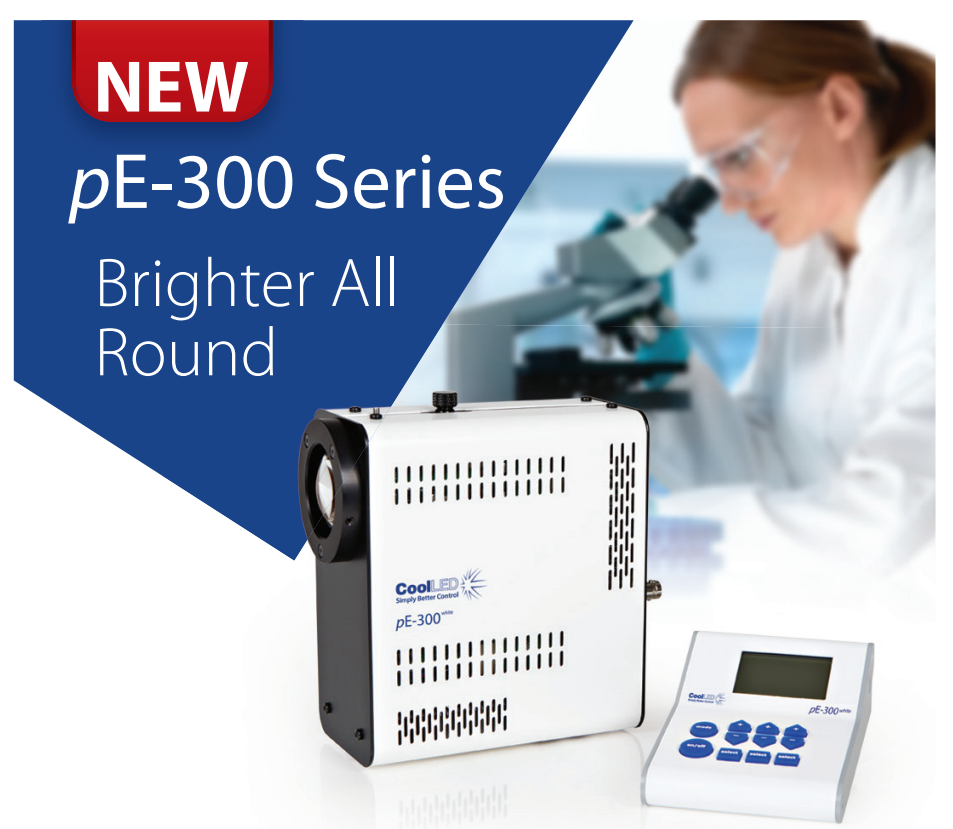

Double the intensity, half the power consumption, quadruple the efficiency

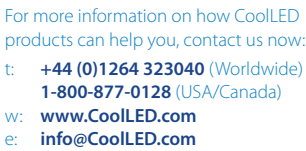

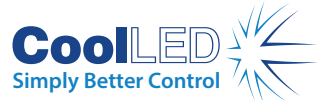

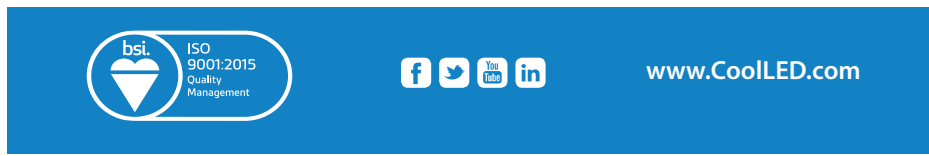

The reconstituted virus was further analyzed by multi-step growth curves using an $\mathrm{MOI}$ of 0.03 in comparison to the parental HCMV strain 40E (Figure 2D). Similar to the parental $40 \mathrm{E}$ strain, RV-TB40-BAC $\mathrm{KL7}_{7}-\mathrm{SE}$ was capable of producing viral titers of $>10^{7}$ infectious units per $\mathrm{mL}$.

Sequence analysis of TB40-BAC $\mathrm{KL}^{-}$ SE showed that the UL128 gene locus required for a broad cell tropism (2,19-22) was intact. To prove that the reconstituted virus is able to infect cell types other than HFFs, we analyzed infection of endothelial cells as a proof-of-principle. As expected, cell-free infections of HUVECs yielded infection efficiencies indistinguishable from the parental virus (Figure 2E).

To facilitate detection of infected cells [e.g., by fluorescence activated cell sorting (FACS)], we attempted to generate a fluorescent version of RV-TB40-BAC $\mathrm{KL}^{-}$ SE. The EGFP gene under the control of the viral major IE promoter/enhancer (MIEP) and with a poly(A) site was introduced at an ectopic position downstream of US34A by markerless mutagenesis. As transcriptional activity is low in this region (17), introduction of additional sequences should not disturb viral gene expression. After transfection of this BAC-DNA into HFFs, fluorescent cells with increasing intensities of EGFP were observed over the following days, but EGFP-expressing cells then showed signs of cell death, and we failed to reconstitute virus from this BAC despite repeated attempts (data not shown). As the MIEP is known for its extraordinary expression strength, and several reports mention a cytotoxic effect of high GFP protein levels (23-26), it is tempting to speculate that premature cell death due to cytotoxic EGFP levels hindered virus growth in our system. Therefore, a 459-bp region of the MIEP enhancer region was deleted to attenuate EGFP expression levels, as described previously (27) (Figure 3A). The resulting RV-TB40-BAC $\mathrm{KL}_{7}-\mathrm{SE}-\mathrm{EGFP}$ virus could be successfully reconstituted within 3 weeks in repeated attempts, indicating that the attenuation of EGFP levels abolished the toxic effect. The reconstituted virus was subsequently analyzed in a one-step growth curve to exclude side effects of the EGFP insertion on virus growth. RV-TB40-BAC $\mathrm{KL}_{7}$-SE-EGFP grew to high titers, similar to the parental $\mathrm{RV}-\mathrm{TB} 40-\mathrm{BAC}_{\mathrm{KL}}-\mathrm{SE}$, with a maximum of 
A
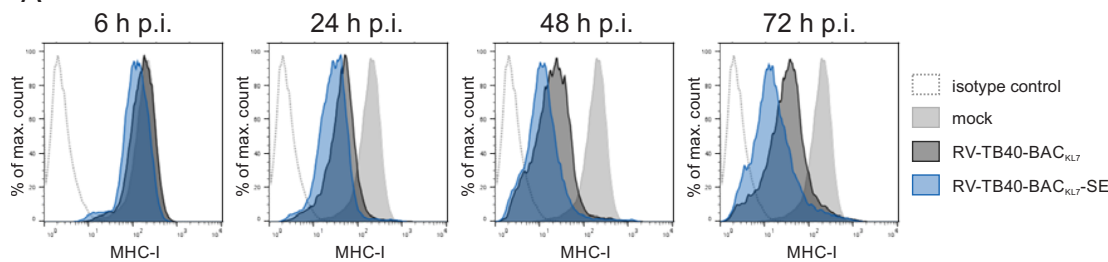

B
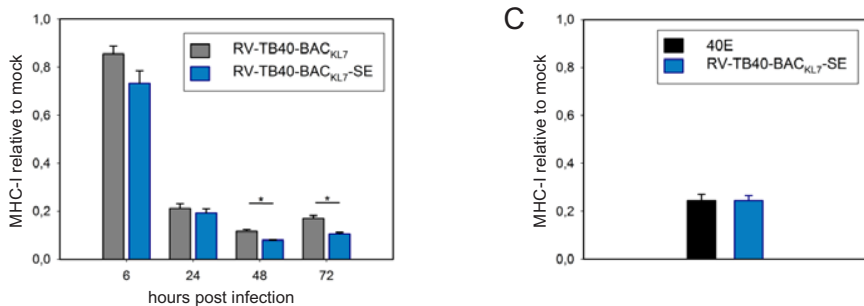

$\mathrm{D}$
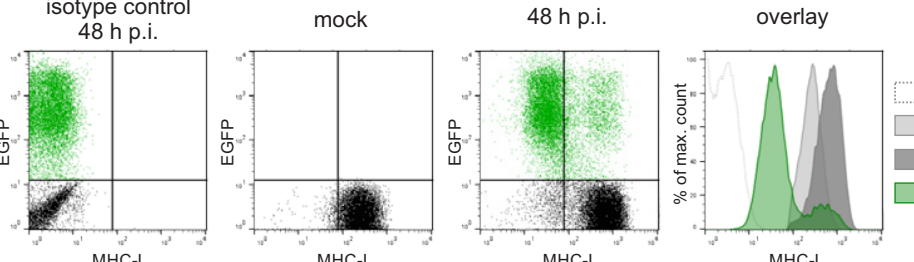

isotype contro

mock

bystander cells

RV-TB40-BAC ${ }_{K 17}$-SE-EGFP

Figure 4. Analysis of MHC-I surface molecules by flow cytometry. (A) Human foreskin fibroblasts (HFFs) were infected with RV-TB40-BAC ${ }_{K L 7}-\mathrm{SE}$ or the parental RV-TB40-BAC ${ }_{K 17}$ at a multiplicity of infection (MOI) of 10. At the indicated time points, cells were detached, stained for MHC-I surface molecules or the respective isotype control antibodies, and analyzed. At all time points tested, RV-TB40$\mathrm{BAC}_{\mathrm{KL}}-\mathrm{SE}$ displayed a more pronounced down-regulation of $\mathrm{MHC}-\mathrm{I}$ molecules as compared with the parental BAC without US2, US3, and US6. Representative histograms of one experiment are shown. (B) Mean values of $\mathrm{MHC}-\mathrm{l}$ expression levels were calculated from geometric mean values obtained in flow cytometry of three independent experiments as described in (A) and are displayed relative to levels of mock-infected cells. Error bars represent standard error of the mean (SEM). Asterisks indicate statistically significant differences ( $t$-test; $P$ value $<0.03$ ). (C) MHC-I surface expression of cells infected with RV-TB40-BAC ${ }_{K 17}$-SE or the parental $40 E$ strain at 3 days post-infection (p.i.). Mean values of three independent experiments were calculated as described in (B). (D) HFFs were infected with RV-TB40-BAC ${ }_{K L 7}-$ SE-EGFP at an MOI of 0.5 to obtain a mixed culture of infected and uninfected cells. At $48 \mathrm{~h}$ p.i., cells were detached, stained for MHC-I surface molecules, and the signals of both EGFP- and APC-stained cells were measured. Only EGFP-expressing cells, representing human cytomegalovirus (HCMV)-infected cells, showed down-regulation of MHC-I surface expression, while non-infected bystander cells showed up-regulation of MHC-I molecules on the cell surface.

$10^{7}$ infectious units per milliliter (Figure 3B). Next, we wondered whether the initiation of EGFP expression from the shortened MIEP would correspond with the initiation of viral gene expression from the natural MIEP. Therefore, the kinetics of EGFP expression was analyzed in comparison to the viral IE proteins pUL122/123, which are driven by the natural MIEP. HFFs were infected with RV-TB40-BAC ${ }_{K L 7}$-SE-EGFP at an $\mathrm{MOI}$ of 50 and fixed at various time points with paraformaldehyde to preserve the natural EGFP fluorescence. The viral pUL122/123 proteins, stained by indirect immunofluorescence, were detectable at $2 \mathrm{~h}$ p.i. EGFP signals became detectable by fluorescence microscopy at $4 \mathrm{~h}$ p.i., and intensity further increased until 72 h p.i., indicating that the EGFP marker is suitable to discriminate infected cells in fluorescence-based readout procedures (Figure 3C).

Finally, we tested the new TB40/Ederivatives for their suitability in FACS analyses (i.e., improved MHC-I downregulation and facilitated detection of infected cells).

As the US2, US3, and US6 proteins are known to inhibit MHC-I surface expression with different kinetics, ranging from immediate-early (US3) to early (US2) and early-late (US6) (28), MHC-I levels on RV-TB40-BAC KLT -SE-infected cells should be decreased compared with

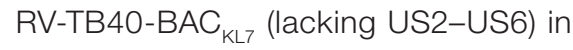
all stages of the HCMV replication cycle. To test this, HFFs were infected with RV-TB40-BAC ${ }_{\mathrm{KL} 7}$ or RV-TB40-BAC $\mathrm{KLT}^{-}$
SE at an MOI of 10, harvested at appropriate time points, and MHC-I surface molecules were stained with a PE-labeled antibody and then analyzed. Indeed, at all time points the down-regulation of MHC-I surface molecules was more pronounced with RV-TB40-BAC ${ }_{K L 7}-\mathrm{SE}$ as compared with the parental virus (Figure $4 \mathrm{~A}$ ), and this difference reached significance in samples at $48 \mathrm{~h}$ and $72 \mathrm{~h}$ p.i. in a set of 3 independent experiments (Figure 4B). As a control, MHC-I surface expression of RV-TB40-BAC $\mathrm{KL}_{\mathrm{T}}$-SEinfected and parental 40E-infected cells were compared at 3 days p.i. to ensure that RV-TB40-BAC ${ }_{K L 7}-S E$ actually reflects the wild-type situation (Figure 4C). Hence, in agreement with the sequencing data showing correct insertion, the functionality of the repaired US2-US6 gene region was also demonstrated. The fact that the parental RV-TB40-BAC ${ }_{K L 7}$ also downregulated $\mathrm{MHC}-\mathrm{I}$ surface molecules to some extent is explained by the presence of the known MHC-I down modulator US11 as well as other potential immune modulators not yet fully characterized, such as US8 and US10 (29).

To test whether the EGFP-expressing version of RV-TB40-BAC $\mathrm{KL}_{7}-\mathrm{SE}$ is suitable for discriminating between infected and uninfected cells by flow cytometry, HFFs were infected with RV-TB40-BAC $\mathrm{KL}^{-}$ SE-EGFP at an MOI of 0.5 to obtain a heterogeneous culture of infected and uninfected cells. At $48 \mathrm{~h}$ p.i., cells were harvested, stained for MHC-I surface molecules with an APC-conjugated antibody, and the signals of the EGFP- and APC-positive cells were measured. The majority of EGFP-positive cells expressed lower levels of MHC-I molecules on the cell-surface (Figure 4D), and the degree of down-regulation was similar to the results obtained with a homogeneous population of infected cells, as analyzed using the parental RV-TB40-BAC ${ }_{K L 7}-\mathrm{SE}$ in Figure 4A. In contrast, the population of uninfected bystander cells lacking EGFP signals showed even higher levels of MHC-I surface staining than uninfected cells. This is in accordance with previous findings, for example, in monocytederived macrophages or dendritic cells, where bystander cells of infected cultures also showed higher levels of $\mathrm{MHC}-1$ surface expression $(30,31)$.

Regarding the genetic repertoire of TB40-BAC $\mathrm{KL}_{7}-\mathrm{SE}$, its sequence was 
compared with the HCMV consensus sequence, and missing or truncated genes are listed in Supplementary Table S2. Similar to the sequence of TB40-BAC4, two genes with immunomodulatory functions are altered (UL40 and UL141), and the additional C-terminal truncation of UL119 could have a functional effect. However, TB40-BAC $\mathrm{KL}_{7}$-SE now provides a suitable basis for the repair of these genes in addition to the restored US gene region, thus enabling comparative studies on HCMV immunomodulatory gene functions. The repaired US genes are functional, as RV-TB40-BAC $\mathrm{KL}_{7}-\mathrm{SE}$ showed a significant decrease of $\mathrm{MHC}-\mathrm{I}$ surface molecules in comparison with the strain lacking US2-US6. Hence, this virus may be useful whenever the complete MHC-I-modulatory effect of wild-type $\mathrm{HCMV}$ is required. The EGFP-expressing variant enables detection of infected cells as early as $4 \mathrm{~h}$ p.i. and may therefore be a preferable option for flow cytometric analyses.

Our aim was to provide a new tool for the scientific community to facilitate HCMV research, particularly immunological studies. Recently, a self-excisable BAC of strain Merlin that maintains the wild-type gene complement of clinical isolates due to conditional repression of RL13 and UL128L was generated (32). This strain, however, releases cell-free virus only when UL128L-and hence endothelial cell tropism -is repressed (9), and even then viral titers are 10 -fold lower than with TB40/E (33). To our knowledge, TB40-BAC $\mathrm{KL}_{7}-\mathrm{SE}$ is the first HCMV BAC clone with an intact USand ULb'-gene region that is capable of producing high titers of cell-free infectivity in the presence of the pentameric $\mathrm{gH} / \mathrm{gL} / \mathrm{pUL} 128 \mathrm{~L}$-complex, which is required for a broad cell tropism. With the complete sequence available, it serves as a tool to generate mutants using common mutagenesis techniques. The conversion of TB40-BAC $\mathrm{KL7}_{\text {T }}$ toward a self-excisable BAC where vector sequences are seamlessly removed provides an advantage for its application in the development of medical products (including vaccines), as additional studies concerning effects of foreign DNA sequences (U.S. Food and Drug Administration, Guidance for industry: considerations for plasmid DNA vaccines for infectious disease indications; https:// doi.org/10.1089/blr.2007.9905) can now be dispensed with.

\section{Author contributions}

K.L.S .and C.S. conceived the study, designed experiments, and drafted the manuscript. K.L.S., A.W., N.S., and Z.W. performed experiments and contributed, together with C.S., to the interpretation of data. All authors worked on correction of the manuscript and approved the final submission.

\section{Acknowledgments}

We thank Karsten Tischer for providing plasmid pEP-MCS-Belo and for his generous and substantial support. We thank Cora Stegmann for critical reading of the manuscript. This work was supported by the Wilhelm-Sander-Foundation (Projekt 2013.002.1 and 2013.002.2).

\section{Competing interests}

The authors declare no competing interests.

\section{References}

1. Boppana, B. and C. Britt. 2013. Synopsis of Clinical Aspects of Human Cytomegalovirus Disease. p. 1-25. In M.J. Reddehase (Ed.), Cytomegaloviruses: From Molecular Pathogenesis to Intervention. Caister Academic Press, London, United Kingdom.

2. Sinzger, C., G. Hahn, M. Digel, R. Katona, K.L. Sampaio, M. Messerle, H. Hengel, U. Koszinowski, et al. 2008. Cloning and sequencing of a highly productive, endotheliotropic virus strain derived from human cytomegalovirus TB40/E. J. Gen. Virol. 89:359368.

3. Yu, D., G.A. Smith, L.W. Enquist, and T. Shenk. 2002. Construction of a self-excisable bacterial artificial chromosome containing the human cytomegalovirus genome and mutagenesis of the diploid TRL/IRL13 gene. J. Virol. 76:2316-2328.

4. Murrell, I., G.S. Wilkie, A.J. Davison, E. Statkute, C.A. Fielding, P. Tomasec, G.W. Wilkinson, and R.J. Stanton. 2016. Genetic stability of bacterial artificial chromosomederived human cytomegalovirus during culture in vitro. J. Virol. 90:3929-3943.

5. Sinzger, C., K. Schmidt, J. Knapp, M. Kahl, R. Beck, J. Waldman, H. Hebart, H. Einsele, and G. Jahn. 1999. Modification of human cytomegalovirus tropism through propagation in vitro is associated with changes in the viral genome. J. Gen. Virol. 80:2867-2877.

6. Hirt, B. 1967. Selective extraction of polyoma DNA from infected mouse cell cultures. J. Mol. Biol. 26:365-369.

7. Borst, E.M., G. Hahn, U.H. Koszinowski, and M. Messerle. 1999. Cloning of the human cytomegalovirus (HCMV) genome as an infectious bacterial artificial chromosome in Esche- richia coli: a new approach for construction of HCMV mutants. J. Virol. 73:8320-8329.

8. Kearse, M., R. Moir, A. Wilson, S. StonesHavas, M. Cheung, S. Sturrock, S. Buxton, A. Cooper, et al. 2012. Geneious Basic: An integrated and extendable desktop software platform for the organization and analysis of sequence data. Bioinformatics 28:1647-1649.

9. Laib Sampaio, K., C. Stegmann, I. Brizic, B. Adler, R.J. Stanton, and C. Sinzger. 2016. The contribution of pUL74 to growth of human cytomegalovirus is masked in the presence of RL13 and UL128 expression. J. Gen. Virol. 97:1917-1927.

10. Tischer, B.K., B.B. Kaufer, M. Sommer, F. Wussow, A.M. Arvin, and N. Osterrieder. 2007. A Self-Excisable Infectious Bacterial Artificial Chromosome Clone of VaricellaZoster Virus Allows Analysis of the Essential Tegument Protein Encoded by ORF9. J. Virol. 81:13200-13208.

11. Wussow, F., H. Fickenscher, B.K. Tischer, M. Gershon, and A. Epstein. 2009. Red-mediated transposition and final release of the mini- $f$ vector of a cloned infectious herpesvirus genome. PLoS One 4:e8178.

12. Tischer, B.K. and B.B. Kaufer. 2012. Viral bacterial artificial chromosomes: Generation, mutagenesis, and removal of mini-F sequences. J. Biomed. Biotechnol. 2012:472537.

13. Yu, D., M.C. Silva, and T. Shenk. 2003. Functional map of human cytomegalovirus AD169 defined by global mutational analysis. Proc. Natl. Acad. Sci. USA 100:12396-12401.

14. Hobom, U., W. Brune, M. Messerle, G. Hahn, and U.H. Koszinowski. 2000. Fast screening procedures for random transposon libraries of cloned herpesvirus genomes: mutational analysis of human cytomegalovirus envelope glycoprotein genes. J. Virol. 74:7720-7729.

15. Dunn, W., C. Chou, H. Li, R. Hai, D. Patterson, V. Stolc, H. Zhu, and F. Liu. 2003. Functional profiling of a human cytomegalovirus genome. Proc. Natl. Acad. Sci. USA 100:14223-14228.

16. Jiang, X.J., B. Adler, K.L. Sampaio, M. Digel, G. Jahn, N. Ettischer, Y.-D. Stierhof, L. Scrivano, et al. 2008. UL74 of human cytomegalovirus contributes to virus release by promoting secondary envelopment of virions. J. Virol. 82:2802-2812.

17. Gatherer, D., S. Seirafian, C. Cunningham, M. Holton, D.J. Dargan, K. Baluchova, R.D. Hector, J. Galbraith, et al. 2011. High-resolution human cytomegalovirus transcriptome. Proc. Natl. Acad. Sci. USA 108:19755-19760.

18. Saleh-Gohari, N. and T. Helleday. 2004. Conservative homologous recombination preferentially repairs DNA double-strand breaks in the S phase of the cell cycle in human cells. Nucleic Acids Res. 32:3683-3688.

19. Wang, D. and T. Shenk. 2005. Human cytomegalovirus virion protein complex required for epithelial and endothelial cell tropism. Proc. Natl. Acad. Sci. USA 102:18153-18158.

20. Ryckman, B.J., M.A. Jarvis, D.D. Drummond, J.A. Nelson, and D.C. Johnson. 2006. Human Cytomegalovirus Entry into Epithelial and Endothelial Cells Depends on Genes UL128 to UL150 and Occurs by Endocytosis and Low-pH Fusion. J. Virol. 80:710-722.

21. Hahn, G., M.G. Revello, M. Patrone, E. Percivalle, G. Campanini, A. Sarasini, M. Wagner, A. Gallina, et al. 2004. Human Cytomegalovirus UL131-128 Genes Are Indis- 
pensable for Virus Growth in Endothelial Cells and Virus Transfer to Leukocytes. J. Virol. 78:10023-10033.

22. Adler, B., L. Scrivano, Z. Ruzcics, B. Rupp, C. Sinzger, and U. Koszinowski. 2006. Role of human cytomegalovirus UL131A in cell typespecific virus entry and release. J. Gen. Virol. 87:2451-2460.

23. Taghizadeh, R.R. and J.L. Sherley. 2008. CFP and YFP, but not GFP, provide stable fluorescent marking of rat hepatic adult stem cells. J. Biomed. Biotechnol. 2008:453590.

24. Liu, H.S., M.S. Jan, C.K. Chou, P.H. Chen, and N.J. Ke. 1999. Is green fluorescent protein toxic to the living cells? Biochem. Biophys. Res. Commun. 260:712-717.

25. Baens, M., H. Noels, V. Broeckx, S. Hagens, S. Fevery, A.D. Billiau, H. Vankelecom, and
P. Marynen. 2006. The dark side of EGFP: Defective polyubiquitination. PLoS One 1:e54.

26. Agbulut, O., A. Huet, N. Niederländer, M. Puceat, P. Menasché, and C. Coirault. 2007. Green fluorescent protein impairs actin-myosin interactions by binding to the actin-binding site of myosin. J. Biol. Chem. 282:10465-10471.

27. Nalaskowski, M.M., P. Ehm, S. Giehler, and G.W. Mayr. 2012. A toolkit for graded expression of green fluorescent protein fusion proteins in mammalian cells. Anal. Biochem. 428:24-27.

28. Chambers, J., A. Angulo, D. Amaratunga, et al. 1999. DNA microarrays of the complex human cytomegalovirus genome: profiling kinetic class with drug sensitivity of viral gene expression. J. Virol. 73:5757-5766.

29. Halenius, A., C. Gerke, and H. Hengel. 2015. Classical and non-classical MHC I molecule

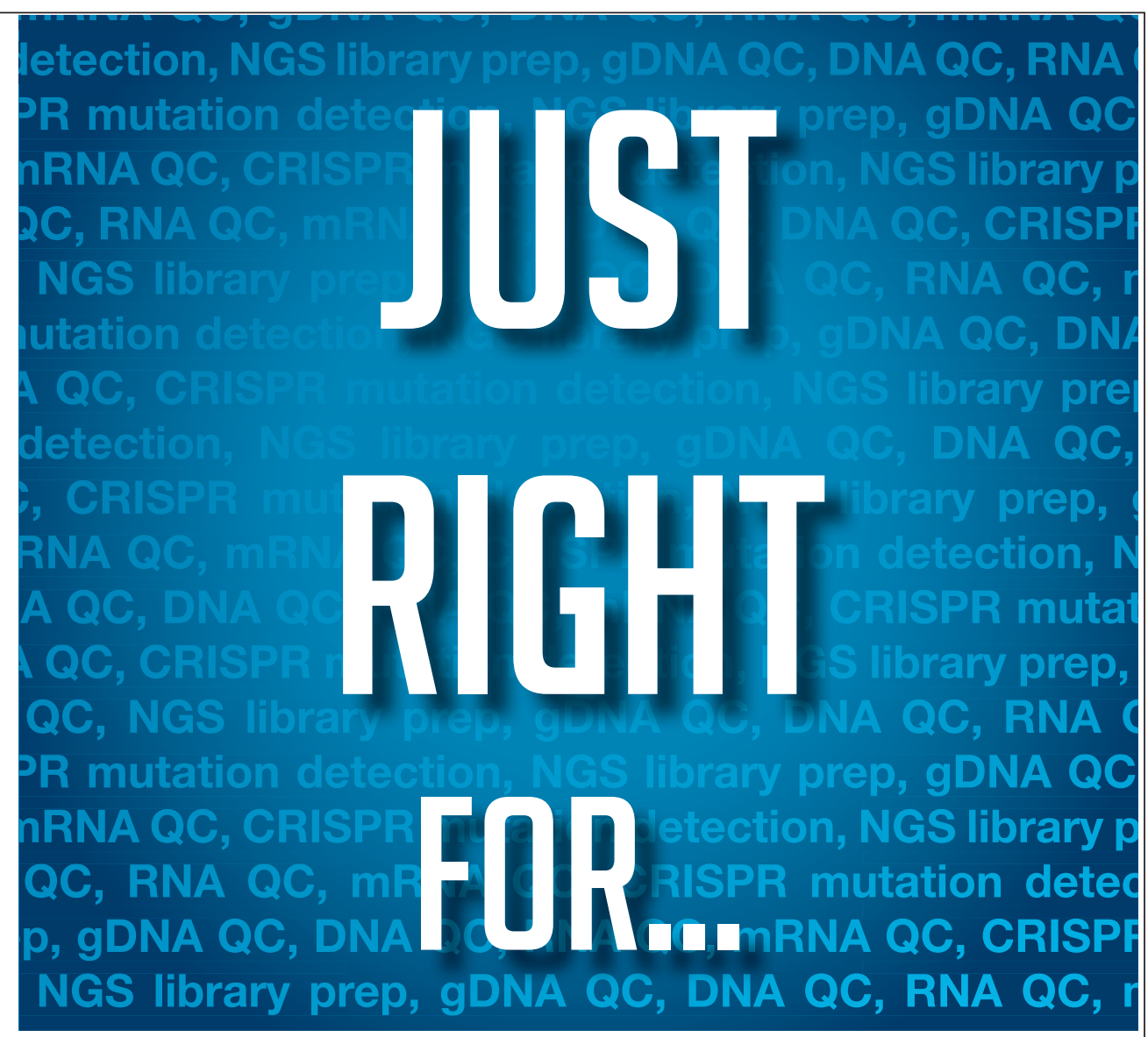

STREAMLINE GENOMIC WORKFLOWS WITH AUTOMATED NUCLEIC ACID QC

The Fragment Analyzer ${ }^{\mathrm{TM}}$ is the premier instrument for automating QC steps in genomics workflow, from raw nucleic acids to final library validation.

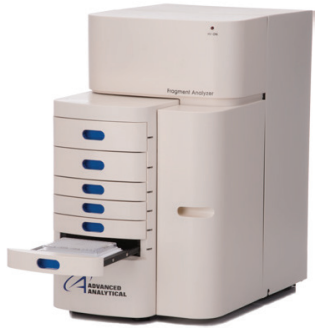

biotechniques@fosterprinting.com

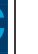

manipulation by human cytomegalovirus: so many targets-but how many arrows in the quiver? Cell. Mol. Immunol. 12:139-153.

30. Bayer, C., S. Varani, L. Wang, P. Walther, S. Zhou, S. Straschewski, M. Bachem, C. Söderberg-Naucler, et al. 2013. Human cytomegalovirus infection of M1 and M2 macrophages triggers inflammation and autologous T-cell proliferation. J. Virol. 87:67-79.

31. Sénéchal, B., A.M. Boruchov, J.L. Reagan, D.N.J. Hart, and J.W. Young. 2004. Infection of mature monocyte-derived dendritic cells with human cytomegalovirus inhibits stimulation of T-cell proliferation via the release of soluble CD83. Blood 103:4207-4215.

32. Stanton, R.J., K. Baluchova, D.J. Dargan, C. Cunningham, O. Sheehy, S. Seirafian, B.P. McSharry, M.L. Neale, et al. 2010. Reconstruction of the complete human cytomegalovirus genome in a BAC reveals $R L 13$ to be a potent inhibitor of replication. J. Clin. Invest. 120:3191-3208

33. Zhou, M., J.-M. Lanchy, and B.J. Ryckman. 2015. Human cytomegalovirus $\mathrm{gH} / \mathrm{gL} / \mathrm{gO}$ promotes the fusion step of entry into all cell types, whereas $\mathrm{gH} / \mathrm{gL} / \mathrm{UL} 128-131$ broadens virus tropism through a distinct mechanism. J. Virol. 89:8999-9009.

34. Keller, M.J., A.W. Wu, J.I. Andrews, P.W. McGonagill, E.E. Tibesar, and J.L. Meier. 2007. Reversal of human cytomegalovirus major immediate-early enhancer/promoter silencing in quiescently infected cells via the cyclic AMP signaling pathway. J. Virol. 81:6669-6681.

Received 10 June 2017; accepted 27 September 2017.

Address correspondence to Kerstin Laib Sampaio, Institut für Virologie, Universität UIm, Albert-Einstein-Allee 11, D-89081 Ulm, Germany. E-mail: kerstin.laib@uni-ulm.de

To purchase reprints of this article, contact: 